\title{
Detection of damaged seeds in laboratory evaluation of precision planter using impact acoustics and artificial neural networks
}

\author{
Hadi Karimi, Hossein Navid, Asghar Mahmoudi \\ Department of Agriculture Machinery, Faculty of Agriculture, Tabriz University, Tabriz, Iran \\ Correspondence: Hadi Karimi. Address: Department of Agriculture Machinery, Faculty of Agriculture, Tabriz University, \\ Tabriz, Iran. Telephone: 98-919-485-3665. Email: hadiekarimi@gmail.com.
}

Received: June 11, 2012

Accepted: August 7, 2012

Published: December 1, 2012

DOI : 10.5430/air.v1n2p67

URL: http://dx.doi.org/10.5430/air.v1n2p67

\begin{abstract}
In the present study, feasibility of laboratory detection of damaged seeds in precision planters caused by malfunction of seed metering device was investigated. An acoustic-based intelligent system was developed for detection of damaged pelleted tomato seeds. To improve the Artificial Neural Network (ANN) models a total of 2000 seeds sound signals, 1000 samples for damaged seeds and 1000 for undamaged ones were recorded. When seed metering device drove out seeds, the ejected seeds were impacted to steel plate, and their acoustic signals were recorded from the impact. The bounced seeds lied on the running grease belt. In each stage of experiments, damaged seeds were determined manually in grease belt and related damaged seed sound signals were designated. Achieved acoustic signals, were processed and potential features were extracted from the analysis of sound signals in time and frequency domains. The method is based on feature generation by Fast Fourier Transform (FFT), feature selection by statistical methods and classification by Multilayer Feed forward Neural Network. Features such as amplitude, phase and power spectrum of sound signals were computed through a 1024-point FFT. By using statistical factors (maximum, minimum, median, mean and variance) for each vector of data, feature vector was reduced to 15 factors. In developing the ANN models, several ANN architectures, each having different numbers of neurons in hidden layer, were evaluated. The best model was chosen after a number of evaluations based on minimizing the mean square error (MSE), correct detection rate (CDR) and correlation coefficient (r). Selected ANN, 15-17-2 was configured for classification. CDR of the proposed ANN model for undamaged and damaged seeds was 99.49 and 100 respectively. MSE of the system was found to be 0.0109 .
\end{abstract}

\section{Key words}

Precision planter, Acoustic, Neural networks, Damaged seeds

\section{I ntroduction}

Optimum plant density and germination rate of seeds are significant factors in crop production. Malfunction of seed metering device in planters may cause seeds to be damaged. Therefore, germination rate and plant density would be changed in the field. In this case, laboratory evaluation of precision planters for identification of seed metering performance is a remarkable object. One of the most frequently used methods for evaluation of the precision planters is grease belt method. Although it is accurate but impose some restriction. The length of the belt limits the data that can be 
taken, and manually determining the damaged seeds is time consuming. Nevertheless, it is convenient and has been tried by some researchers for evaluating one row seed metering mechanism ${ }^{[1]}$. In one of the latest techniques, Kocher et al. ${ }^{[2]}$ and Lan et al. ${ }^{[3]}$ used a seed detector system comprising 24 photo transistors. Results obtained by Panning et al. ${ }^{[4]}$ through this system showed inaccuracy of the opto-electronic sensor system was related to seeds with less than 3 millimeters in diameter. Navid et al. ${ }^{[5]}$ concluded that identification broken seeds were not available by image processing in laboratory evaluation of seed metering because the image-processing algorithm is based on color. Recently, acoustical experiments have been carried out in detection and classification of agricultural products. Pearson ${ }^{[6]}$ used discrimination analysis on data acquired from sound signal sampling in the time domain, for separation of open and closed shell Kerman variety of pistachio nuts. The classification accuracy of this method was about $97 \%$. Acoustic impact method used by Hosainpour, et al. ${ }^{[7]}$ to be discriminate between potato tubers and clods. The accuracy and capacity of this technique were reported about $97 \%$ and 20 ton/hr., respectively. Artificial neural network (ANN) is a simplified model of a real neural system, and like a brain can learn by processing on practical data, networks learn general rules by calculating numerical data or examples of that, there are called intelligent systems. Neural networks can learn from data directly because they do not need statistical properties so, it is an important advantage of these networks ${ }^{[8]}$. Neural network predicts output with its corresponding input, without considering any initial supposition and previous knowledge of relations among studied parameters ${ }^{[9]}$. Artificial neural networks (ANN) offer much faster and more flexible approach in classification fields. In addition, in noisy obscure pattern, ANN models are more efficient than statistical pattern classifiers. Mahmoudi ${ }^{[10]}$ used artificial neural networks (ANNs) to detect walnut varieties and correct detection rate of the proposed ANN model for two walnut genotypes, were 99.64 and 96.56. This study was done to investigate the feasibility of using impact sound signals for detection of damaged seeds in laboratory evaluation of seed metering device. An artificial neural network was used as a decision-making unit. For this purpose, potential features were extracted from analysis of emitted sound signals.

\section{Material and method}

The planter used in this research, was designed in the agricultural machinery department, at University of Tabriz. It was designed for pelleted tomato seeds (sun f1) seeding. Its seed-metering device was 15-cell vertical- roller type with $118 \mathrm{~mm}$ diameter. The planter was installed on the $11 \mathrm{~m}$ long, $40 \mathrm{~cm}$ wide grease belt. The detecting system of damaged seeds consisted of an impact plate, an acoustic unit and a PC based data-acquisition for evaluating system efficiency. An impact plate fixed at the $30^{\circ}$ angle right under the metering device so that each seed would impact one time to the plate. This angle was determined by trial and error. Falling distance from the end point of the metering device to the impact plate was fixed to $20 \mathrm{~cm}$. Preliminarily experimental results showed that steel plates proved better than glass or wood for separating seeds. The impact plate was made up by a polished block of stainless steel about $10 \times 10 \times 0.6 \mathrm{~mm}$. A microphone (VM-034CY model), sensitive to frequencies up to $100 \mathrm{kHz}$, was used to capturing impact sound signals. To eliminate the environmental noise effects, the microphone was installed inside an isolated chamber. To prevent the chamber from acoustical reflections, it was filled with glass wool. Microphone output was sent to a PC based data-acquisition where it was digitized using a sound card (Intel ${ }^{8} 82801$ BA/BAM AC`97Audio controller) at a sampling frequency of $44.1 \mathrm{kHz}$, with 16 bit resolution. The personal computer was used for acquiring, saving and processing of the data. Whole planter test rig is illustrated in figure 1 and a schematic diagram describing the detection system is shown in Figure 2. A parallel hardware and software architecture was used to perform the described duty. As it was shown in figure 1 the test rig was devised so that, the falling seeds from metering device impacted to the plate one by one and lied on the grease belt respectively. The grease belt was used to determine which impacted seeds were damaged. In each stage of experiments, the metering device was run up until the first seed on the grease belt reached to the end of the belt, then each damaged and undamaged seeds on the belt were signed and relating sound signals were designated. The experiment was continued until 2000 impact signals from damaged and undamaged seeds were acquired. The sound signals were acquired by the microphone, digitized by the sound card and saved by using the Matlab data acquisition toolbox ${ }^{[11]}$. Since the maximum frequency of the sound card was $44.1 \mathrm{kHz}$ and data acquisition continued for $15.87 \mathrm{~ms}$. After triggering, upon getting a 
trigger signal the computer acquired 700 data points from every sample in the time-domain. Matlab software was used for data collection and management.

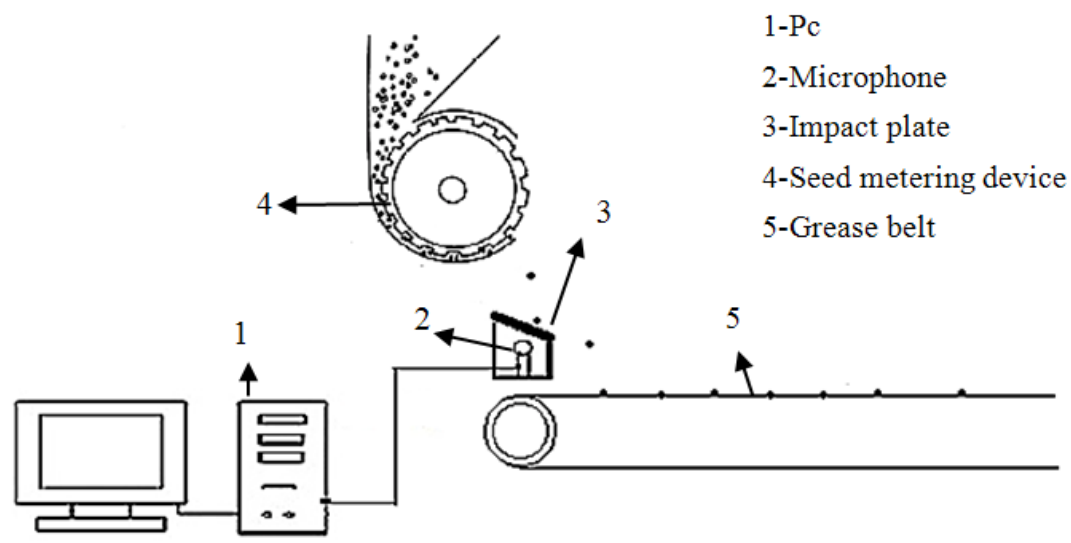

Figure 1. Whole planter rig test

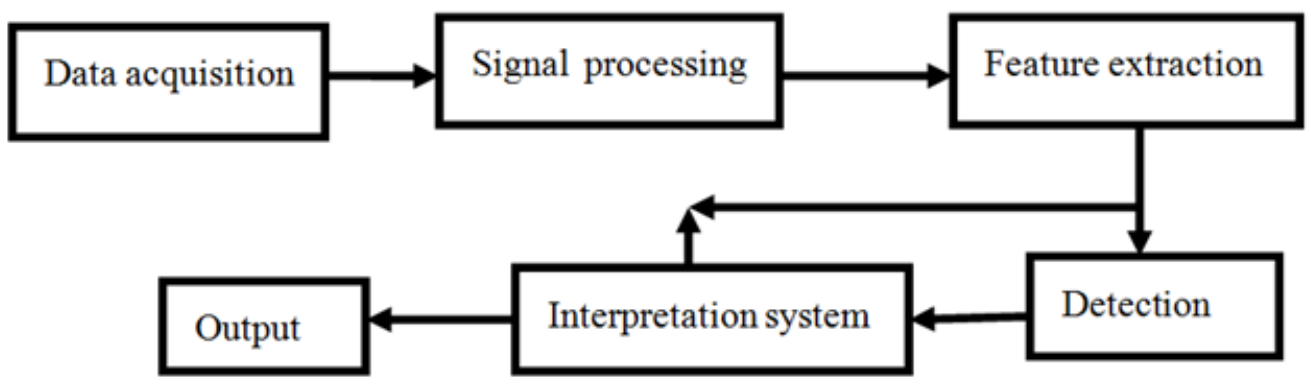

Figure 2. The block diagram of the detection system

\section{Signal processing and feature extraction}

From impact sound of the seeds, 2000 data were collected, 1000 for damaged pelleted tomato seeds and 1000 for undamaged ones. To extracting potential features, recorded sound signals were processed and analyzed in time and frequency domains. Typical emitted sound signals from undamaged seeds and damaged one are shown in Figure 3, 4. Although the maximum peak values of various undamaged seed's sound signals were slightly larger than damaged ones, the preliminary attempts to use only time-domain features were not successful. However, in order not to lose any useful transient features, all 700 data point amplitude (Amp) values were considered as features. A 1024-point fast Fourier transform (FFT) was computed from each sound signal. Magnitude, power spectral density (PSD) and phase angle of each spectrum were computed according to symmetry of sound signal in Frequency domain. Only 512 data from 1024 data point were used for calculating PSD and phase. To disclose the differences between two types of seeds, for an instance fifteenth PSD data from 512 data point was illustrated in figure 5. The first thousands are undamaged seeds, and the others are the damaged ones. For each seed 1724 features were attained. For real time systems, the dimension of the input vector is too large, so we diminished the dimension of the input vectors. In this case, we achieved five statistical factors (maximum, minimum, median, mean and variance) for each vector of amplitude, power spectral density and phase angle. 


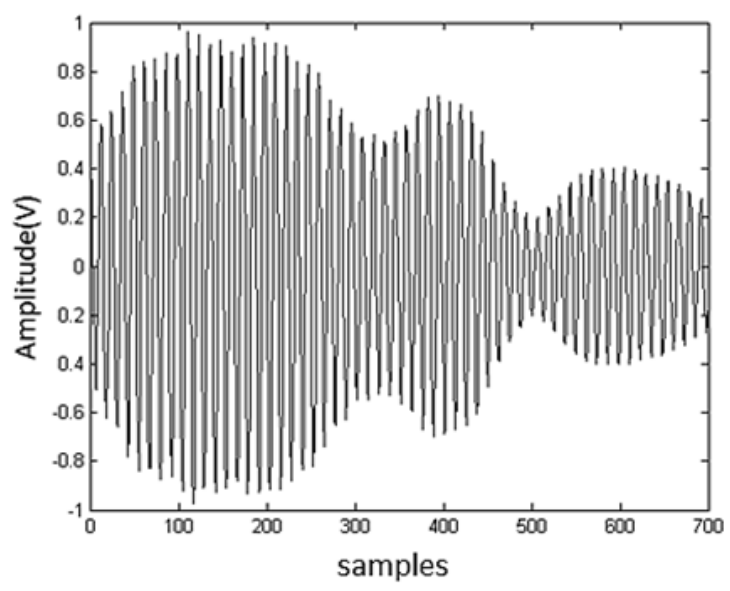

Figure 3. Impact signal from typical undamaged pelleted tomato seed

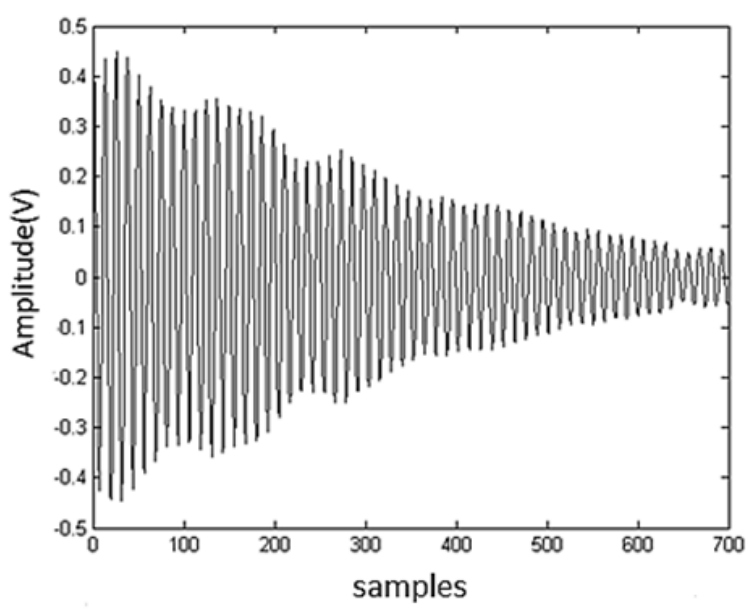

Figure 4. Impact signal from typical damaged pelleted tomato seed

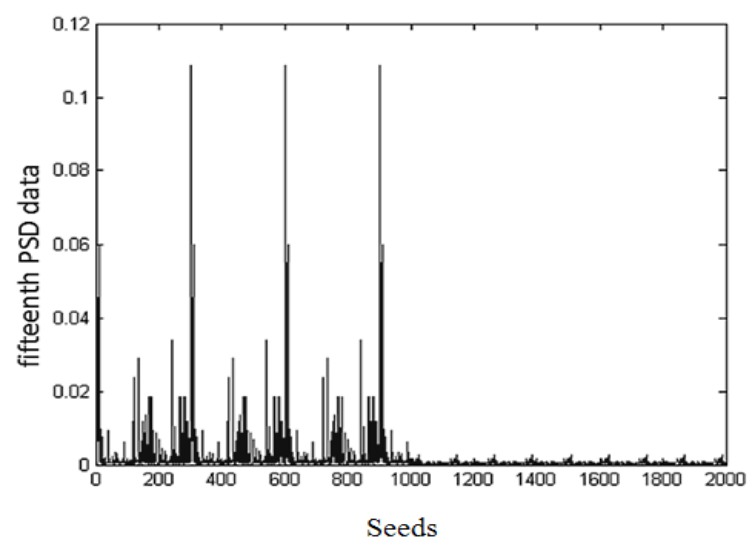

Figure 5. PSD for fifteenth data of 2000 seeds 


\section{Artificial neural networks}

Although many types of neural networks can be used for classification purposes, in this study we used multilayer perceptrons (MLPs) which are the most widely studied and used neural network classifiers. MLPs are normally trained with the back propagation algorithm. In fact, the renewed interest in ANNs was in part triggered by the emergence of back propagation learning rule. The back propagation rule propagates the errors through the network and allows adaptation of the hidden processing elements (PEs). Two important characteristics of MLP are (i) use of nonlinear PEs such as logistic or hyperbolic tangent and (ii) their massive interconnectivity, i.e. any element of a given layer feeds all the elements of the next layer. The MLP is trained with error correction learning, which means that the desired response for the system must be known a priori. After adequate training, the network weights are adapted and employed for cross validation in order to determine the ANN model overall performance. Gradient descent with momentum (GDM) learning rule is an improvement to the straight GD rule in the sense that a momentum term is used to speed up learning and stabilizing convergence. Therefore, the GDM method of learning is used throughout this study. To minimize ANN training time, only one hidden layer was considered. If the number of hidden neurons is too small, the model will not be flexible enough to model the data well. On the other hand, if there are too many layers, the model will over fit the data. For training process, we picked a network parameter to vary. The best network weights are saved at the parameter variation, run, and epoch when the cross validation error is minimum (see Table 1) number of neuron in hidden layer was determined using an exhaustive search from 1 to 20 nodes. The Neural Network with 17 nodes in hidden layer (see Figure 6) had the least standard deviation error as well as high stability. In developing ANN models, the linear function of the input layer and the non-linear hyperbolic tangent function at hidden and output layer were used as transfer functions. Learning rate was 0.7 throughout the momentum-learning rule. As an added guard against over-fitting, the data sets were divided into three randomly selected data sets; $65 \%$ of data were used for training, $15 \%$ for testing and the remaining $20 \%$ were used for cross validation. NeuroSolutions 5.0 software was used for designing and testing of ANN models.

Table 1. Parameters of best training for neural networks

\begin{tabular}{lll}
\hline Best Networks & Training & Cross Validation \\
\hline Hidden 1 PEs & 20 & 17 \\
Run \# & 3 & 1 \\
Epoch \# & 1000 & 1000 \\
Minimum MSE & 0.00840975 & 0.010991521 \\
Final MSE & 0.00840975 & 0.010991521 \\
\hline
\end{tabular}

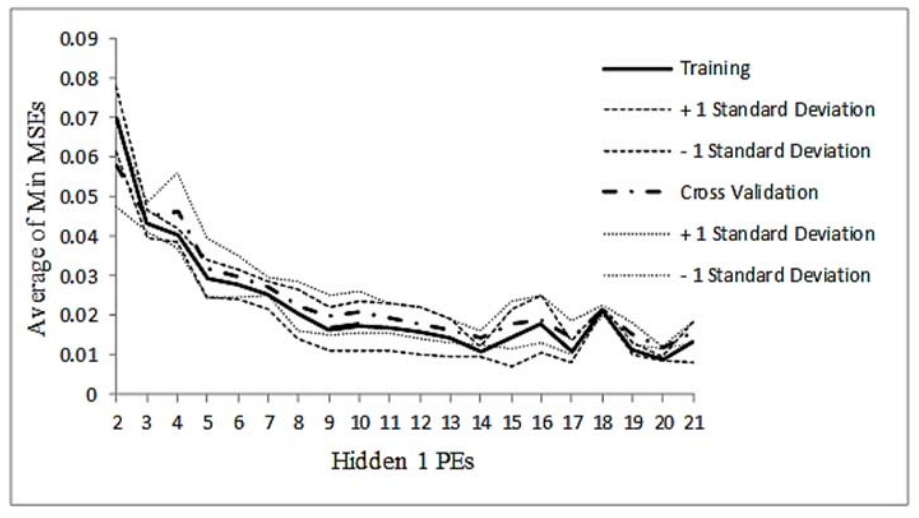

Figure 6. Average of minimum MSEs with standard deviation boundaries 


\section{Analysis result}

Different combination of potential statistical features were configured to ANN and obtained models were tested (see Table 2). Different designed models were compared based on mean square error (MSE), correlation coefficient (r) and correct detection rate. The formula used for determining MSE is:

$$
\operatorname{MSE}=\frac{1}{N P} \sum_{j=0}^{P} \sum_{i=0}^{N}\left(D_{i j}-Y_{i j}\right)^{2}
$$

Table 2. Combinations of statistical features were selected and tested by neural networks

\begin{tabular}{|c|c|c|c|c|c|c|}
\hline \multirow[t]{2}{*}{ Features } & \multicolumn{2}{|c|}{$\begin{array}{l}\text { Correct detection rate } \\
(\mathrm{CDR}) \%\end{array}$} & \multicolumn{2}{|c|}{$\begin{array}{l}\text { Correlation coefficient } \\
\text { (r) }\end{array}$} & \multicolumn{2}{|c|}{$\begin{array}{l}\text { Mean square Error } \\
\text { ( MSE) }\end{array}$} \\
\hline & $\begin{array}{l}\text { Undamaged } \\
\text { seeds }\end{array}$ & $\begin{array}{l}\text { Damaged } \\
\text { seeds }\end{array}$ & $\begin{array}{l}\text { Undamaged } \\
\text { seeds }\end{array}$ & $\begin{array}{l}\text { Damaged } \\
\text { seeds }\end{array}$ & $\begin{array}{l}\text { Undamaged } \\
\text { seeds }\end{array}$ & Damaged seeds \\
\hline Amplitude & 87.14 & 91.57 & 0.81 & 0.81 & 0.0831 & 0.0833 \\
\hline PSD & 87.62 & 94.94 & 0.84 & 0.84 & 0.0711 & 0.0718 \\
\hline Angle & 91.78 & 96.89 & 0.89 & 0.89 & 0.0491 & 0.0488 \\
\hline Amplitude\&PSD\&Angle & 99.49 & 100 & 0.98 & 0.98 & 0.0072 & 0.0074 \\
\hline Amplitude\&Angle & 97.27 & 97.27 & 0.94 & 0.94 & 0.0265 & 0.0264 \\
\hline Amplitude\&PSD & 91.75 & 99.02 & 0.92 & 0.91 & 0.0381 & 0.0396 \\
\hline PSD\&Angle & 98.37 & 100 & 0.97 & 0.97 & 0.0111 & 0.0114 \\
\hline
\end{tabular}

Where $\mathrm{P}$ is the number of output PEs, $\mathrm{N}$ is the number of exemplars in the data set, $Y_{i j}$ is network output for exemplar $\mathrm{i}$ at processing element $\mathrm{j}$ and $D_{i j}$ is desired output for exemplar $\mathrm{i}$ at processing element $\mathrm{j}$. The amplitudes, PSD and phase angle features individually were not enough potential to discriminate between damaged tomato pellet and undamaged ones. In summary, the best combination was amplitudes with PSD and phase angle features. The final structure of network was 15 input nodes, 17 hidden nodes and two output nodes. Table 3 and Table 4 shows the performance of selected ANN model in the case of determining damaged and undamaged pelleted tomato seeds.

Table 3. Performance of optimal ANN configuration

\begin{tabular}{lcc}
\hline Performance & undamaged & damaged \\
\hline MSE & 0.007216339 & 0.007402363 \\
R & 0.985831111 & 0.985515008 \\
Percent Correct & 99.49238579 & 100 \\
& & \\
\hline
\end{tabular}

Table 4. Evaluation of correct detection between damaged and undamaged pelleted tomato seeds

\begin{tabular}{lll}
\hline Output / Desired & undamaged & damaged \\
\hline undamaged & 196 & 0 \\
damaged & 1 & 203 \\
\hline
\end{tabular}

Also Network Module Sensitivity About the Mean was done. This testing process provides a measure of the relative importance among the inputs of the neural model and illustrates how the model output varies in response to variation of an input (see figure 7). 


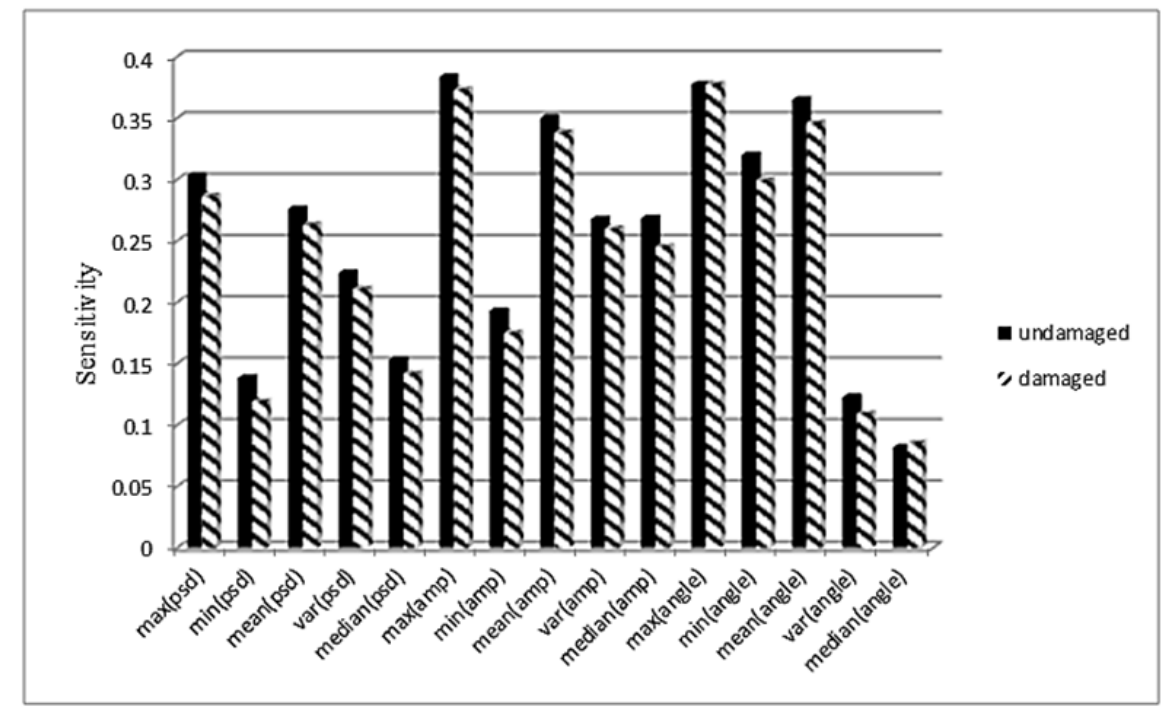

Figure 7. Network Module Sensitivity about the Mean

\section{Conclusion}

Laboratory methods in this research have been developed and used for evaluation of seed metering device performance in the field of detecting damaged seeds. Detection system, based on the combination of acoustic detection and statistical factors facilitated ANN classifier was devised. Optimal neural network for detection exhibited a 15-17-2 structure, with 17 neurons in its hidden layer. Testing reports showed that correct detection rate (CDR) for this model was 99.49 and 100 percent for undamaged pelleted tomato seeds and damaged ones respectively. Up until now, no successful automatic system for detecting damaged seeds in laboratory evaluation of precision planter has been reported. With regard to advantages of this system such as high accuracy, large capacity and practicality, small space requirement and low cost of equipment and computation, This system can be used to recognize other seed's varieties in different kind of seed metering devices and can be used instead of grease belt method in laboratory evolution of precision planters.

\section{References}

[1] Singh R C, Singh G, Saraswat D C. Optimization of design and operational parameters of a pneumatic seed metering device for planting cottonseeds. Biosystems Engineering. 2005; 92(4): 429-438. http://dx.doi.org/10.1016/j.biosystemseng.2005.07.002

[2] Kocher M F, Lan Y, Chen C, Smith J A. Opto-electronic sensor system for rapid evaluation of planter seed spacing uniformity. Trans. ASAE.1998; 41(1): 237-245.

[3] Lan Y, Kocher M F, Smith J A. Opto-electronic sensor for laboratory measurement of planter seed spacing with small seeds. Journal of Agricultural Engineering Research.1999; 72(2): 119-127. http://dx.doi.org/10.1006/jaer.1998.0353

[4] Panning J W, Kocher M F, Smith J A, Kachman S D. Laboratory and field testing of seed spacing uniformity for sugar beet planters. Applied Engineering in Agriculture. 2000; 16(1): 7-13.

[5] Navid H, Ebrahimian S, Gassemzadeh H R, Mousavinia M J. Laboratory Evaluation of Seed Metering Device Using Image Processing Method [online]. Australian journal of agricultural Engineering. 2011; 2: 1-4.

[6] Pearson T C. Detection of pistachio nuts with closed shells using impact acoustics. Applied Engineering in Agriculture. 2001; 17(2): 249-253.

[7] Hosainpour A, Komarizade M H, Mahmoudi A, Shayesteh M G. High speed detection of potato and clod using an acoustic based intelligent system. Expert Systems with Applications. 2011. http://dx.doi.org/10.1016/j.eswa.2011.02.164

[8] Vakil-Baghmisheh M T. Farsi Character Recognition Using Artificial Neural Networks [PhD Thesis]. Faculty of Electrical Engineering, University of Ljubljana; 2002. 
[9] Torrecilla J S, Otero L, Sanz P D. Neural networks approach for thermal/pressure food processing. Food Engineering. 2004; 62: 89-95. http://dx.doi.org/10.1016/S0260-8774(03)00174-2

[10] Mahmoudi A. Development of a Suitable Algorithm Using Artificial Neural Networks for Sorting of Pistachio Nuts with Closed Shells Using Impact Acoustics [PhD thesis]. Department of Agricultural Machinery, University of Tehran, Karaj, Iran. $2006 ; 144$.

[11] MathWorks, MATLAB User's Guide, The MathWork, Inc.; 2010.

[12] Omid M, Mahmoudi A, Omid M H. Development of pistachio sorting system using principal component analysis (PCA) assisted artificial neural network (ANN) of impact acoustics. Expert Systems with Applications. 2010; 37(10): 7205-7212. http://dx.doi.org/10.1016/j.eswa.2010.04.008

[13] Simin k, Mahmoud A, Hosainpour A. Detection of Walnut Varieties Using Impact Acoustics and Artificial Neural Networks (ANNs). Modern Applied Science. 2012; 6(1). 